\title{
Polymorphisms Associated with Age at Onset in Patients with Moderate-to-Severe Plaque Psoriasis
}

\author{
Rocío Prieto-Pérez, ${ }^{1}$ Guillermo Solano-López, ${ }^{2}$ Teresa Cabaleiro, ${ }^{1,3}$ \\ Manuel Román, ${ }^{1}$ Dolores Ochoa, ${ }^{1}$ María Talegón, ${ }^{1}$ Ofelia Baniandrés, ${ }^{4}$ \\ José Luis López-Estebaranz, ${ }^{5}$ Pablo de la Cueva, ${ }^{6}$ \\ Esteban Daudén, ${ }^{2}$ and Francisco Abad-Santos ${ }^{1,3}$ \\ ${ }^{1}$ Clinical Pharmacology Service, Hospital Universitario de la Princesa, Instituto Teófilo Hernando, \\ Instituto de Investigación Sanitaria Princesa (IP), Universidad Autónoma de Madrid (UAM), 28006 Madrid, Spain \\ ${ }^{2}$ Dermatology Service, Hospital Universitario de la Princesa, Instituto de Investigación Sanitaria Princesa (IP), 28006 Madrid, Spain \\ ${ }^{3}$ Centro de Investigación Biomédica en Red de Enfermedades Hepáticas y Digestivas (CIBERehd), Instituto de Salud Carlos III, \\ Madrid, Spain \\ ${ }^{4}$ Dermatology Service, Hospital Universitario Gregorio Marañón, 28007 Madrid, Spain \\ ${ }^{5}$ Dermatology Service, Hospital Universitario Fundación Alcorcón, 28922 Madrid, Spain \\ ${ }^{6}$ Dermatology Service, Hospital Universitario Infanta Leonor, 28031 Madrid, Spain
}

Correspondence should be addressed to Francisco Abad-Santos; francisco.abad@salud.madrid.org

Received 18 June 2015; Accepted 15 September 2015

Academic Editor: Fulvia Ceccarelli

Copyright ( 2015 Rocío Prieto-Pérez et al. This is an open access article distributed under the Creative Commons Attribution License, which permits unrestricted use, distribution, and reproduction in any medium, provided the original work is properly cited.

\begin{abstract}
Psoriasis is a chronic skin disease in which genetics play a major role. Although many genome-wide association studies have been performed in psoriasis, knowledge of the age at onset remains limited. Therefore, we analyzed 173 single-nucleotide polymorphisms in genes associated with psoriasis and other autoimmune diseases in patients with moderate-to-severe plaque psoriasis type I (earlyonset, $<40$ years) or type II (late-onset, $\geq 40$ years) and healthy controls. Moreover, we performed a comparison between patients with type I psoriasis and patients with type II psoriasis. Our comparison of a stratified population with type I psoriasis $(n=155)$ and healthy controls $(N=197)$ is the first to reveal a relationship between the CLMN, FBXL19, CCL4L, C17orf51, TYK2, IL13, SLC22A4, CDKAL1, and HLA-B/MICA genes. When we compared type I psoriasis with type II psoriasis $(N=36)$, we found a significant association between age at onset and the genes PSORS6, TNF- $\alpha$, FCGR2A, TNFR1, CD226, HLA-C, TNFAIP3, and CCHCR1. Moreover, we replicated the association between rs12191877 (HLA-C) and type I psoriasis and between type I and type II psoriasis. Our findings highlight the role of genetics in age of onset of psoriasis.
\end{abstract}

\section{Introduction}

Psoriasis is a chronic inflammatory skin disorder with a major genetic component. The prevalence of chronic plaque psoriasis is around $2 \%$ in the general population [1]. The many genetic studies performed in recent years showed that genes such as interleukin 23 receptor (IL23R) and IL12B and tumor necrosis factor alpha $(T N F \alpha)$ are closely associated with psoriasis and related diseases such as rheumatoid arthritis, psoriatic arthritis, and Crohn's disease [2]. Human leukocyte antigen $\mathrm{C}(\mathrm{HLA}-\mathrm{C})^{*} 0602$ is the allele most closely associated with this disease [3].

The age at onset of psoriasis follows a bimodal distribution [4]: type I psoriasis appears before the age of 40 years (early-onset), with a peak at 16-22 years; type II psoriasis appears after the age of 40 years (late-onset), with a peak at 57-60 years [5]. Type I psoriasis has been associated with several single-nucleotide polymorphisms (SNPs) in genes associated with the immune response (Table 1). For example, HLA-C 0602 is more strongly associated with type I psoriasis 


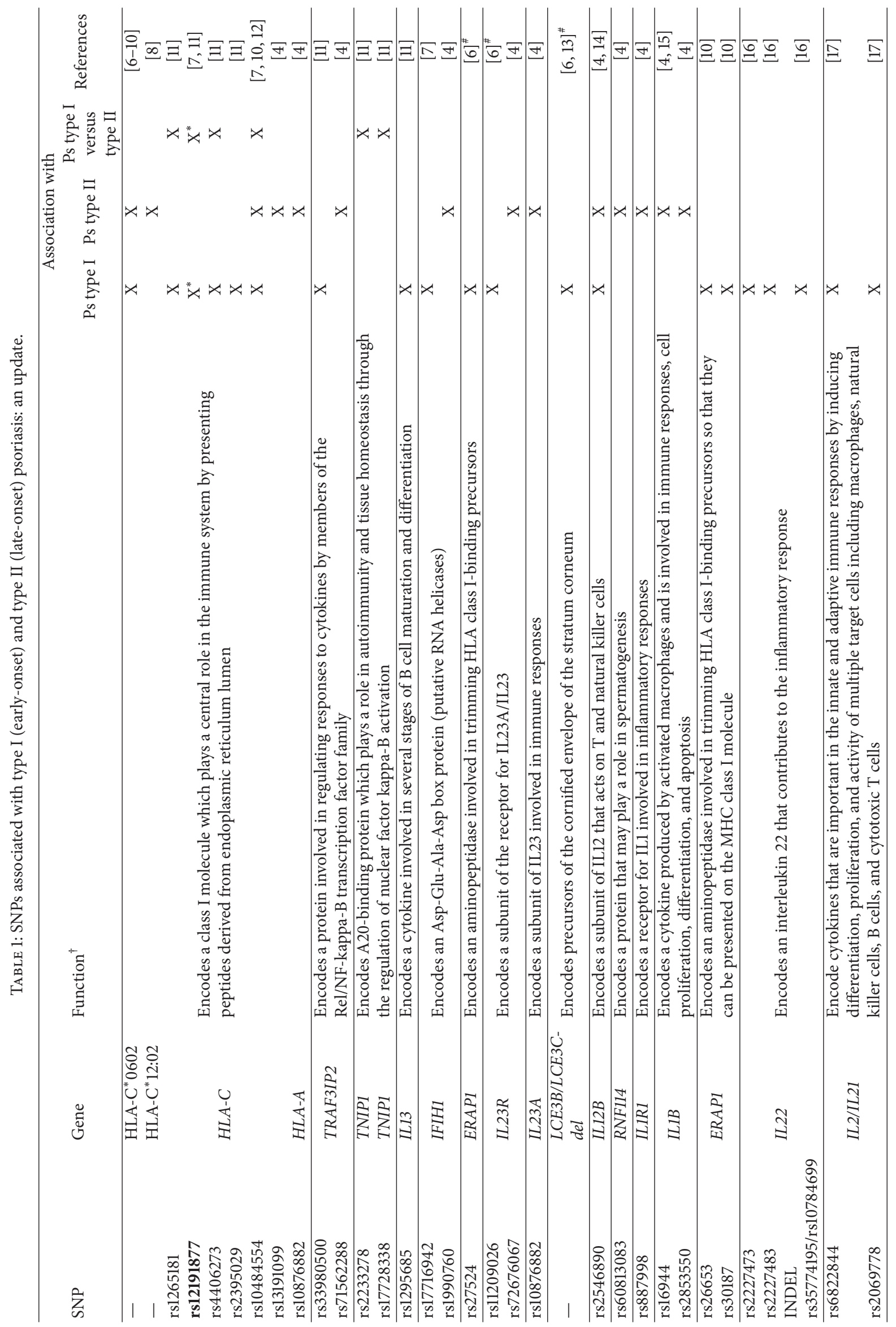




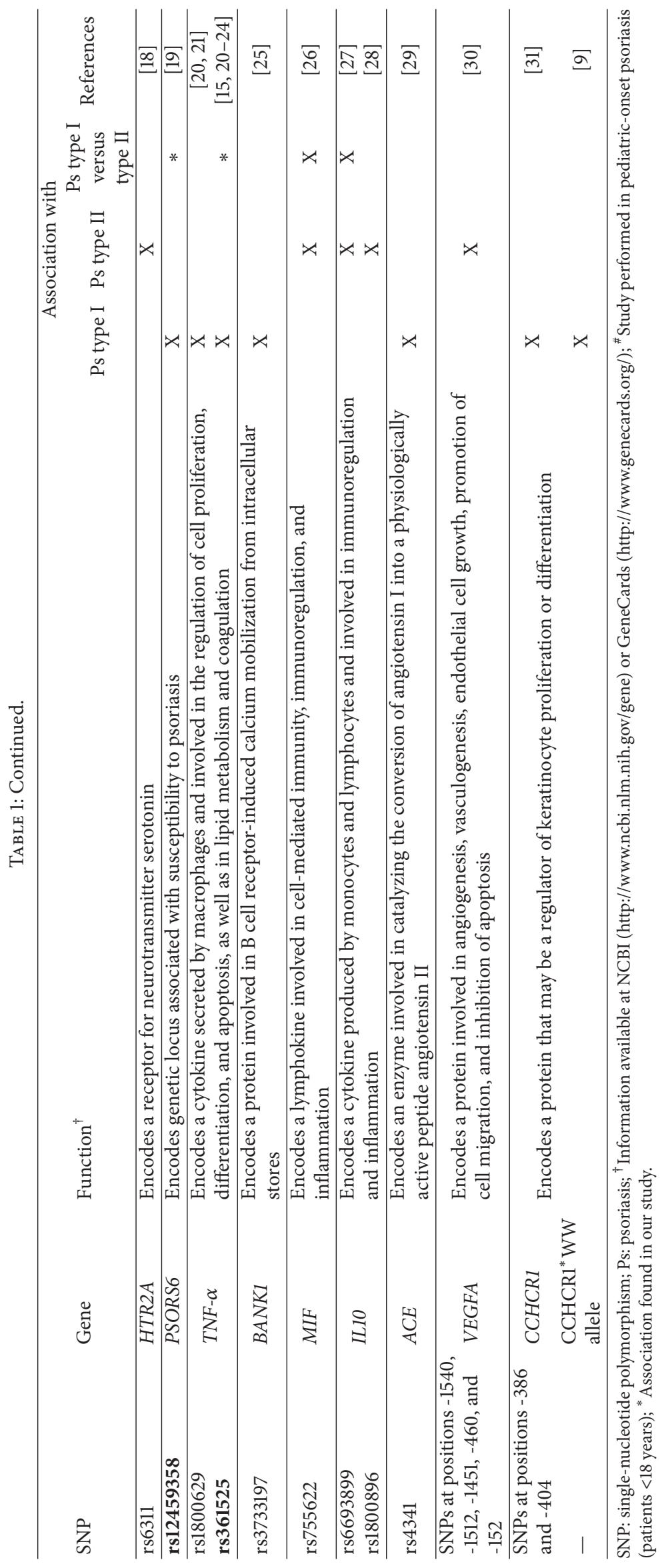


than with type II psoriasis [5]. Although several association studies have already been performed in psoriasis in both populations (type I or type II psoriasis patients), knowledge of age at onset remains limited and controversial (Table 1) [6]. Therefore, we performed a candidate gene study, where we evaluated genetic susceptibility to type I or type II psoriasis in patients with moderate-to-severe chronic plaque psoriasis. This approach may help us to identify SNPs previously associated with psoriasis or other autoimmune diseases [2] that are specific to type I or type II psoriasis. Furthermore, our genetic study could improve our understanding of psoriasis and of its etiology and pathogenesis.

\section{Material and Methods}

2.1. Experimental Design. We recruited 198 Caucasian patients with moderate-to-severe plaque type psoriasis (psoriasis area and severity index $>10$ ) who attended the department of dermatology in four university hospitals in Madrid between 16/10/2007 and 17/12/2012. Five samples did not fulfill the quality criteria of the Human Genotyping Unit-CeGen (CEGEN, Spanish National Cancer Research Centre, Madrid, Spain), and 2 samples had insufficient volume. We also included 197 healthy volunteers (controls) recruited between 10/01/2011 and 14/12/2012 from the Clinical Pharmacology Service (Hospital Universitario de la Princesa, Madrid, Spain). All the volunteers were Caucasian and had no personal or family history of psoriasis (at least 2 generations).

The protocol fulfilled Spanish law on biomedical research and was approved by the Ethics Committee for Clinical Investigation of Hospital Universitario de la Princesa. All controls and patients gave their written informed consent to donate a sample for investigation. The samples are kept in the Clinical Pharmacology Service.

2.2. Selection of the Polymorphisms. We preselected 320 SNPs based on an extensive review of 449 articles describing the association between polymorphisms and psoriasis and response to biological drugs and psoriasis and related inflammatory diseases (rheumatoid arthritis, psoriatic arthritis, and Crohn's disease) [2]. We finally selected 192 SNPs based on minor allele frequency $(\geq 0.05)$ and on the results of studies performed in Caucasians and psoriatic patients. Information on the 173 SNPs analyzed can be found in supplementary Table S1, which is published in [3].

2.3. Sample Processing. A 3-mL peripheral blood sample was extracted from each subject in EDTA tubes. DNA was obtained from samples using an automatic DNA extractor (MagNa Pure System, Roche Applied Science, USA) and its concentration was quantified in Nanodrop ND-1000 Spectrophotometer (Wilmington, USA). The extracted DNA was stored at $-80^{\circ} \mathrm{C}$ in the Clinical Pharmacology Service until use.

2.4. Genotyping. A total of 196 samples from patients (2 samples of 198 cases had insufficient volume) and 197 samples from controls were sent to the Human Genotyping UnitCeGen to genotype 192 SNPs. The analysis was performed using the Illumina Veracode genotyping platform. If fluorescence was low or the genotype clusters were undifferentiated, the SNPs were removed. In addition, if the call rate was less than $95 \%$ of the average of the 192 SNPs analyzed, the samples were removed. Since CEGEN quality criteria were not met in 19 SNPs and 5 patients, we finally analyzed 173 SNPs in 191 patients and 197 controls.

2.5. Statistical Analysis. The statistical analysis was performed to compare the following stratified populations: patients with type I psoriasis $(N=155)$ or type II psoriasis $(N=36)$ versus controls $(N=197)$ and patients with type I psoriasis versus cases with type II psoriasis. Hardy-Weinberg equilibrium was tested for all the SNPs analyzed using the SNPStats program [32]. Allele and genotype frequencies were also calculated using the SNPStats program. SNPs that were not in Hardy-Weinberg equilibrium in controls were removed from the subsequent analysis [33].

The univariate analysis was performed using $\mathrm{R}$ 3.0.2. (SNPassoc) [34]. We constructed various logistic regression models depending on the main types of inheritance (codominant, dominant, recessive, and additive). In the additive model, the presence of 2 mutant alleles confers double the risk of 1 mutant allele [33]. The results were adjusted for rs 12191877 (SNP that is strongly associated with the HLA-C ${ }^{*} 0602$ allele and is highly prevalent in our population) $[3,35]$. The optimal model was selected using the lower Akaike Information Criterion (AIC). Subsequently, SNPs with $p<0.1$ in the univariate analysis (adjusted for rs12191877) were included in a multivariate logistic regression model to adjust for relevant confounding factors (SPSS 15.0). The results of the univariate analysis were adjusted for rs12191877, except when we compared patients with type I psoriasis and patients with type II psoriasis (the influence of rs12191877 was not very relevant). We expressed the results as the odds ratio (OR), $95 \%$ confidence interval, and $p$ value.

\section{Results}

3.1. Study Population. The study population included 155 patients with moderate-to-severe chronic plaque type I psoriasis (92 men and 63 women), 36 patients with type II psoriasis (19 men and 17 women), and 197 controls (98 men and 99 women). The mean age was $46.01 \pm 13.11$ years in patients with type I psoriasis $(45.72 \pm 11.69$ in men and $46.43 \pm 15.04$ in women), $67.72 \pm 11.85$ years in patients with type II psoriasis $(65.95 \pm 11.18$ in men and $69.71 \pm 12.59$ in women $)$, and $24.51 \pm 4.29$ years in the controls $(25.07 \pm 4.94$ in men and $23.95 \pm 3.46$ in women). The mean age at onset of psoriasis was $23.31 \pm 8.52$ in patients with type I psoriasis and $52.58 \pm 10.45$ in patients with type II psoriasis. Analysis of the effect of sex on our results revealed no significant association.

3.2. Genotyping Results. A total of 192 SNPs were analyzed (see supplementary Table S1 published in [3]). However, only 173 SNPs fulfilled the quality criteria. One SNP was monomorphic (rs165161 in the JUNB gene) and was excluded from the statistical analysis. The genotyping success rate was $89.82 \%$, and the reproducibility rate was $100 \%$. 
TABLE 2: Results of univariate linear regression analysis (unadjusted and adjusted for rs12191877 in $H L A-C$ ) and multivariate linear regression analysis (155 patients with type I psoriasis versus 197 controls). In the multivariate analysis, we included the SNPs with $p<0.1$ in the univariate analysis adjusted for HLA-C. Only polymorphisms that were significant in the multivariate analysis are shown.

\begin{tabular}{|c|c|c|c|c|c|c|c|c|c|}
\hline \multirow[t]{2}{*}{ SNP } & \multirow[t]{2}{*}{ Gene } & \multirow[t]{2}{*}{ Model } & \multirow{2}{*}{$\begin{array}{l}\text { Risk } \\
\text { genotype }\end{array}$} & \multicolumn{2}{|c|}{$\begin{array}{l}\text { Univariate unadjusted. Type I } \\
\text { patients versus controls }\end{array}$} & \multicolumn{2}{|c|}{$\begin{array}{l}\text { Univariate adjusted for } \\
\text { HLA-C }\end{array}$} & \multicolumn{2}{|l|}{ Multivariate } \\
\hline & & & & OR $(95 \% \mathrm{CI})$ & $p$ value & OR (95\% CI) & $p$ value & OR $(95 \% \mathrm{CI})$ & $p$ value \\
\hline rs2282276 & CLMN & $\mathrm{A}$ & & $1.74(0.96-3.15)$ & 0.066 & $1.95(1.04-3.65)$ & 0.037 & $3.90(1.13-13.3$ & 0.031 \\
\hline rs10782001 & FBXL19 & A & GG/AG & $1.58(1.13-2.21)$ & 0.007 & $1.59(1.09-2.32)$ & 0.016 & $2.10(1.05-4.17)$ & 0.035 \\
\hline rs1634517 & $C C L 4 L$ & $\mathrm{D}$ & $\mathrm{AA} / \mathrm{AC}$ & $0.89(0.58-1.36)$ & 0.590 & $0.64(0.39-1.05)$ & 0.073 & $0.34(0.14-0.84)$ & 0.019 \\
\hline rs1975974 & C17orf51 & A & GG/AG & $0.80(0.57-1.14)$ & 0.220 & $0.66(0.44-0.99)$ & 0.040 & $0.48(0.23-0.99)$ & 0.048 \\
\hline rs12720356 & TYK2 & A & GG/GT & $0.42(0.21-0.81)$ & 0.019 & $0.27(0.13-0.58)$ & 0.0003 & $0.10(0.03-0.39)$ & 0.001 \\
\hline rs1800925 & IL13 & $\mathrm{R}$ & $\mathrm{TT}$ & $0.18(0.02-1.45)$ & 0.051 & $0.17(0.02-1.49)$ & 0.061 & $0.01(0.00-0.73)$ & 0.034 \\
\hline rs3792876 & SLC22A4 & A & $\mathrm{TT} / \mathrm{CT}$ & $1.57(0.89-2.76)$ & 0.110 & $1.87(0.98-3.55)$ & 0.057 & 3.75 (1.19-11.83) & 0.024 \\
\hline rs6908425 & CDKAL1 & A & $\mathrm{TT} / \mathrm{CT}$ & $0.67(0.47-0.97)$ & 0.029 & $0.58(0.39-0.89)$ & 0.01 & $0.41(0.20-0.85)$ & 0.017 \\
\hline rs12191877 & $H L A-C$ & A & $\mathrm{TT} / \mathrm{CT}$ & $5.92(3.83-9.15)$ & $2.50 E-19$ & - & - & $30.54(10.62-87.85)$ & 0.000 \\
\hline rs13437088 & HLA-B/MICA & $\mathrm{D}$ & $\mathrm{TT} / \mathrm{CT}$ & $2.17(1.42-3.34)$ & $3.00 E-04$ & $1.93(1.19-3.13)$ & 0.007 & $2.52(1.01-6.31)$ & 0.048 \\
\hline
\end{tabular}

CLMN: calponin-like transmembrane gene; FBXL19: F-box and leucine-rich repeat protein 19; CCL4L: chemokine (C-C motif) ligand 4-like; C17orf51: chromosome 17 open reading frame 51; TYK2: nonreceptor tyrosine-protein kinase; IL13: interleukin 13; SLC22A4: solute carrier family 22 member 4; CDKAL1: cyclin-dependent kinase 5 regulatory subunit associated protein 1-like 1; HLA: major histocompatibility complex; MICA: major histocompatibility complex class I polypeptide-related sequence A; SNPs: single-nucleotide polymorphisms; OR: odds ratio of presenting type 1 psoriasis; CI: confidence interval; A: additive; R: recessive; D: dominant; -: no data.

All the minor allele frequencies were in Hardy-Weinberg equilibrium except 9 SNPs in the controls and 12 SNPs in the patients (see supplementary Table S1 published in [3]). The 9 SNPs which were not in Hardy-Weinberg equilibrium in controls were removed from the statistical analysis [33].

3.3. Association with Type I or Type II Psoriasis. Our findings showed an association between type I psoriasis and 10 SNPs $(N=155$ versus $N=197$ controls): rs1634517 (CCL4L), rs1975974 (C17orf51), rs12720356 (TYK2), rs1800925 (IL13), and rs6908425 (CDKAL1) decreased the risk of psoriasis 2.94fold, 2.08-fold, 10-fold, 100-fold, and 2.44-fold, respectively; and rs2282276 (CLMN), rs10782001 (FBXL19), rs3792876 (SLC22A4), rs12191877 (HLA-C), and rs13437088 (HLA$B / M I C A$ ) increased the risk of psoriasis 3.90 -fold, 2.10-fold, 3.75-fold, 30.54-fold, and 2.52-fold, respectively (Table 2). However, comparison of 36 patients with type II psoriasis and 197 controls revealed no significant association (results not shown).

Four SNPs were associated with significant decreases in the risk of type I psoriasis $(N=155)$ compared with type II psoriasis $(N=36)$, namely, rs191190 (TNFR1; 126.08-fold), rs361525 (TNF- $\alpha$; 190.76-fold), and rs10499194 and rs6920220 (TNFAIP3; 155.02-fold and 19.14-fold, resp.). We also found 5 SNPs that were associated with a significant increase in the risk of type I psoriasis, namely, rs1801274 (FCGR2A; 5.26fold), rs763361 (CD226; 33.3-fold), rs12459358 (PSORS6; 11.11fold), rs12191877 (HLA-C; 12.5-fold), and rs1576 (CCHCR1; 166.66-fold) (Table 3).

\section{Discussion}

About $75 \%$ of patients with chronic plaque psoriasis have type I psoriasis before age 40 [4], whereas a lower number of patients develop psoriasis at around 50-60 years [11]. Our results are consistent with these findings, since $79.06 \%$ of our patients developed psoriasis before the age of 40 .

When we compared patients with type I psoriasis and controls, we found 10 significant SNPs in CLMN, FBXL19, CCL4L, C17orf51, TYK2, IL13, SLC22A4, CDKAL1, HLA-C, and HLA-B/MICA.

The HLA-C* 0602 allele is a risk factor for psoriasis [35] and has been associated with both type I [6-9] and type II psoriasis [10]. In one study, $85.3 \%$ of patients with type I psoriasis had this allele [5], whereas only $14.7 \%$ of patients with type II psoriasis were carriers [5]. Other authors found an association between rs10484554 (HLA-C) and type I psoriasis compared with type II psoriasis $(\mathrm{OR}=3.24$ in type I) [12]. rs10484554 has also been associated with type II psoriasis [10]. In a recent GWAS, the $H L A-C$ gene was associated with type I psoriasis $(p=2.97 E-18$ for rs1265181, $p=2.58 E-15$ for rs12191877, $p=1.84 E-15$ for rs4406273, and $p=1.10 E-07$ for rs2395029), but not with type II psoriasis after application of the Bonferroni correction [11]. In addition, our results showed significant differences in rs12191877 (HLA-C) in patients with type I psoriasis $(p=$ $2.50 E-19)$. However, we did not find this association in patients with type II psoriasis, probably owing to the small sample size in this group $(N=36)$.

Munir et al. found an association between rs1295685 in the IL13 gene and type I psoriasis ( $p=2.47 E-03)$ [11]. Our results showed an association between another SNP in IL13 (rs1800925) and type I psoriasis $(p=0.034)$. In addition, Munir et al. did not obtain significant results when they compared controls with type II psoriasis or type I psoriasis with type II psoriasis [11]. Both SNPs in IL13 have been associated with predisposition to psoriasis $[36,37]$.

Our comparison of patients with type I psoriasis and controls is the first to obtain significant results for a series 
TABLE 3: Results of univariate and multivariate linear regression analyses (155 patients with psoriasis type I versus 36 cases with psoriasis type II). SNPs with $p<0.1$ in the univariate analysis were included in the multivariate analysis. Only polymorphisms that were significant in the multivariate analysis are shown.

\begin{tabular}{|c|c|c|c|c|c|c|c|}
\hline \multirow{2}{*}{ SNP } & \multirow{2}{*}{ Gene } & \multirow{2}{*}{ Model } & \multirow{2}{*}{ Risk genotype } & \multicolumn{2}{|c|}{ Univariate Ps patients type I versus type II } & \multicolumn{2}{|c|}{ Multivariate } \\
\hline & & & & OR $(95 \% \mathrm{CI})$ & $p$ value & OR (95\% CI) & $p$ value \\
\hline rs1801274 & FCGR2A & A & $\mathrm{CC} / \mathrm{CT}$ & $1.96(1.12-3.45)$ & 0.016 & $5.26(1.11-25)$ & 0.037 \\
\hline rs191190 & TNFR1 & $\mathrm{D}$ & $\mathrm{CC} / \mathrm{CT}$ & $0.43(0.17-1.11)$ & 0.065 & $0.01(1.44 E-04-0.44)$ & 0.018 \\
\hline rs763361 & $C D 226$ & $\mathrm{D}$ & $\mathrm{TT} / \mathrm{CT}$ & $2.08(0.99-4.35)$ & 0.056 & $33.33(1.11-1000)$ & 0.043 \\
\hline rs12459358 & PSORS6 & A & $\mathrm{TT} / \mathrm{CT}$ & $2.44(1.32-4.55)$ & 0.002 & $11.11(1.32-100)$ & 0.026 \\
\hline rs10499194 & TNFAIP3 & $\mathrm{D}$ & $\mathrm{TT} / \mathrm{CT}$ & $0.38(0.17-0.90)$ & 0.02 & $0.01(6.77 E-05-0.61)$ & 0.030 \\
\hline rs12191877 & $H L A-C$ & $\mathrm{~A}$ & $\mathrm{TT} / \mathrm{CT}$ & $2.33(1.23-4.35)$ & 0.006 & $12.50(1.06-100)$ & 0.045 \\
\hline rs6920220 & TNFAIP3 & A & $\mathrm{AA} / \mathrm{AG}$ & $0.55(0.30-1.03)$ & 0.068 & $0.05(0.003-0.90)$ & 0.042 \\
\hline rs361525 & $T N F-\alpha$ & $\mathrm{C}$ & $\mathrm{AG}$ & $2.17(0.62-7.69)$ & 0.087 & $0.01(5.48 E-05-0.50)$ & 0.024 \\
\hline rs1576 & CCHCR1 & $\mathrm{D}$ & GG/GC & $2.56(1.22-5.26)$ & 0.012 & $166.67(2.32-1000)$ & 0.019 \\
\hline
\end{tabular}

FCGR2A: Fc fragment of IgG low affinity IIa receptor; TNFR1: tumor necrosis factor receptor 1; CD226: CD226 antigen; PSORS6: psoriasis susceptibility 6; TNFAIP3: tumor necrosis factor alpha-induced protein 3; HLA-C: major histocompatibility complex; TNFAIP3: tumor necrosis factor alpha-induced protein 3; TNF- $\alpha$ : tumor necrosis factor alpha; CCHCR1: coiled-coil alpha-helical rod protein 1; SNPs: single-nucleotide polymorphisms; OR: odds ratio of presenting type I psoriasis; CI: confidence interval; A: additive; D: dominant; C: codominant.

of SNPs in type I psoriasis, although the SNPs have already been associated with the risk of psoriasis. rs10782001 in FBXL19 [38], rs1975974 in C17orf51 [38], rs12720356 in TYK2 $[3,39]$, rs3792876 in SLC22A4 [3], rs6908425 in CDKAL1 [40], and rs13437088 in HLA-B/MICA [35] have previously been associated with psoriasis, but not with type I psoriasis.

Furthermore, SNPs in CLMN (rs2282276) and CCL4L (rs1634517) have not been associated with psoriasis or age at onset.

We found no significant differences between patients with type II psoriasis and controls owing to the small sample size $(N=36)$.

Comparison between patients with type I psoriasis and patients with type II psoriasis revealed significant associations for the following genes: FCGR2A, TNFR1, CD226, PSORS6, TNFAIP3, HLA-C, TNF- $\alpha$, and CCHCR1.

Polymorphisms in CCHCR1 (-386 and -404 , CCHCR ${ }^{*} \mathrm{WW}$ allele) have been associated with type I psoriasis [9, 31]. We found significant differences between rs1576 in CCHCR1 and age at onset. In a study comparing controls (54.8\%) and patients with psoriasis type II (66.0\%), Allen et al. showed a significant increase in the number of patients carrying rs1576 [41]. This SNP has been associated with psoriasis elsewhere [42].

Douroudis et al. analyzed rs763361 in CD226 in patients with early-onset psoriasis and patients with late-onset psoriasis, although they found no associations [43]. We performed the same analyses and found significant differences between the groups. In addition, rs763361 in CD226 has been associated with severity of psoriasis [43].

rs12459358 in PSORS6 has been associated with type I psoriasis ( $\mathrm{G}$ risk allele, $\mathrm{OR}=1.47$ and $p=0.005$ ) [19]. In contrast, our data showed an association between the $\mathrm{T}$ allele and type I psoriasis $(\mathrm{OR}=11.11 ; p=0.026)$. rs361525 (-238) in the TNF $\alpha$ gene has been associated with susceptibility to psoriasis [44], and the A allele was more frequent in male patients with type I psoriasis $(p=$ $2 E-07)[15,22]$. We found significant results in rs361525 $(T N F-\alpha)$ when we compared patients with type I psoriasis and patients with type II psoriasis, although we found no gender differences. Other authors confirmed our association with type I psoriasis in Caucasian [20, 23] and Mongolian patients [24]. A meta-analysis showed an association between rs361525 and type I psoriasis [21]. Baran et al. found no significant differences between rs1800629 in the -308 promoter (TNF $\alpha)$ and type I or type II psoriasis [45].

Likewise, rs12191877 in HLA-C has been associated with increased risk of psoriasis [35]. Munir et al. [11] compared patients with type I psoriasis and patients with type II psoriasis and obtained significant results for rs1265181, rs4406273, and rs12191877 in HLA-C. We replicated these results in rs12191877 ( $\mathrm{T}$ allele risk; $p=0.045$ ).

rs191190 in TNFR1 [46] and rs10499194 in TNFAIP3 [3] have been associated with psoriasis, but not with age of onset. Moreover, rs1801274 in FCGR2A and rs6920220 in TNFAIP3 have not been studied in patients with psoriasis according to age of onset. Given the small sample size in the group with type II psoriasis in our study, our results should be interpreted with caution.

Our results highlight the role of the immune system in psoriasis and enhance our understanding of pathogenic mechanisms. Such knowledge can help to optimize treatment.

Our study is subject to a series of limitations. First, mean age varied between the cases and the controls. Second, the sample size was limited by the number of study patients treated in the dermatology department, thus making it difficult to detect SNPs with a low probability of causing 
psoriasis. Third, since the SNPs were selected based on a literature review, several major SNPs may not yet have been investigated.

In conclusion, our study confirmed an association between rs12191877 (HLA-C) and type I psoriasis and between type I and type II psoriasis patients. Ours is the first study to show an association between CLMN, FBXL19, CCL4L, C17orf51, TYK2, IL13, SLC22A4, CDKAL1, and $H L A-B / M I C A$ and type I psoriasis. Moreover, CLMN and CCL $4 L$ have not been previously described in psoriasis. In addition, PSORS6 and TNF $\alpha$ have been described as more prevalent genes in type I psoriasis and we showed a significant association when we compared type I psoriasis and type II psoriasis. Ours is the first study to identify an association between FCGR2A, TNFR1, CD226, TNFAIP3, and CCHCR1 and age at onset of psoriasis. Our results suggest that genetics could play a role in age at onset. However, further studies are needed to confirm our findings.

\section{Conflict of Interests}

E. Daudén has potential conflict of interests (advisory board member, consultant, grants, research support, participation in clinical trials, honoraria for speaking, and research support) with the following pharmaceutical companies: AbbVie (Abbott), Amgen, Janssen-Cilag, Leo Pharma, Novartis, Pfizer, MSD, and Celgene. F. Abad-Santos has been a consultant or investigator in clinical trials sponsored by the following pharmaceutical companies: Abbott, Alter, Chemo, Faes, Farmalíder, Ferrer, GlaxoSmithKline, JanssenCilag, Kern, Normon, Servier, Teva, and Zambon. P. de la Cueva has conflict of interests (advisory board member, consultant, grants, research support, participation in clinical trials, honoraria for speaking, and/or research support) with the following pharmaceutical companies: AbbVie (Abbott), Astellas, Janssen-Cilag, Leo Pharma, Novartis, Pfizer, MSD, Gebro, Isdin, and Lilly. J. L. López Estebaranz has conflict of interests (advisory board member, speaker, or participation in clinical trials) with AbbVie, Amgen, Pfizer, MSD, Janssen-Cilag, Lilly, Celgene. Ofelia Baniandrés has conflict of interests (participation in clinical trials and honoraria for speaking) with the following pharmaceutical companies: AbbVie (Abbott), Janssen-Cilag, Leo Pharma, Pfizer, and MSD.

\section{Authors' Contribution}

Rocío Prieto-Pérez and Guillermo Solano-López contributed equally to the paper.

\section{Acknowledgments}

This work was supported by Instituto de Salud Carlos III (FIS PI10/01740), Fundación Teófilo Hernando, and AbbVie. Rocío Prieto-Pérez has a grant from University Autónoma de Madrid (FPI Program 2013). The authors are grateful to Thomas O'Boyle for editorial assistance and to Cristina Carazo for helping to set up the patient databases.

\section{References}

[1] V. Chandran, "Genetics of psoriasis and psoriatic arthritis," Indian Journal of Dermatology, vol. 55, no. 2, pp. 151-156, 2010.

[2] R. Prieto-Pérez, T. Cabaleiro, E. Daudén, and F. Abad-Santos, "Gene polymorphisms that can predict response to anti-TNF therapy in patients with psoriasis and related autoimmune diseases," Pharmacogenomics Journal, vol. 13, no. 4, pp. 297-305, 2013.

[3] R. Prieto-Pérez, G. Solano-López, T. Cabaleiro et al., "New immune system genetic polymorphisms associated with moderate-to-severe plaque psoriasis: a case-control study," British Journal of Dermatology, vol. 172, no. 5, pp. 1432-1435, 2015.

[4] H. Hébert, J. Bowes, R. Smith et al., "Identification of loci associated with late-onset psoriasis using dense genotyping of immune-related regions," British Journal of Dermatology, vol. 172, no. 4, pp. 933-939, 2015.

[5] T. Henseler and E. Christophers, "Psoriasis of early and late onset: characterization of two types of psoriasis vulgaris," Journal of the American Academy of Dermatology, vol. 13, no. 3, pp. 450-456, 1985.

[6] J. G. M. Bergboer, A. M. Oostveen, M. E. A. de Jager et al., "Paediatric-onset psoriasis is associated with ERAP1 and IL23R loci, LCE3C_LCE3B deletion and HLA-C*06," British Journal of Dermatology, vol. 167, no. 4, pp. 922-925, 2012.

[7] Y. Lu, S. Kane, H. Chen et al., "The role of 39 psoriasis risk variants on age of psoriasis onset," ISRN Dermatology, vol. 2013, Article ID 203941, 4 pages, 2013.

[8] T. Mabuchi, T. Ota, Y. Manabe et al., "HLA-C" 12:02 is a susceptibility factor in late-onset type of psoriasis in Japanese," Journal of Dermatology, vol. 41, no. 8, pp. 697-704, 2014.

[9] Y.-T. Chang, H. N. Liu, Y. M. Shiao et al., "A study of PSORS1C1 gene polymorphisms in Chinese patients with psoriasis," British Journal of Dermatology, vol. 153, no. 1, pp. 90-96, 2005.

[10] J. Lysell, L. Padyukov, I. Kockum, P. Nikamo, and M. Ståhle, "Genetic association with ERAP1 in psoriasis is confined to disease onset after puberty and not dependent on HLA-C* 06 ," Journal of Investigative Dermatology, vol. 133, no. 2, pp. 411-417, 2013.

[11] S. Munir, S. B. Rahman, S. Rehman et al., "Association analysis of GWAS and candidate gene loci in a Pakistani population with psoriasis," Molecular Immunology, vol. 64, no. 1, pp. 190-194, 2015.

[12] S. Masouri, I. Stefanaki, K. P. Kypreou et al., "Replication of risk variants for psoriasis in a Southern European case-control study: correlation with clinical subphenotypes," British Journal of Dermatology, vol. 173, no. 2, pp. 552-554, 2015.

[13] L. Xu, Y. Li, X. Zhang et al., "Deletion of LCE3C and LCE3B genes is associated with psoriasis in a northern Chinese population," British Journal of Dermatology, vol. 165, no. 4, pp. 882-887, 2011.

[14] O. Haase, H. Mosaad, M. A. Eldarouti et al., “TNFAIP3 and IL12B gene polymorphisms associated with psoriasis vulgaris in an Egyptian cohort," Journal of the European Academy of Dermatology and Venereology, vol. 29, no. 7, pp. 1297-1301, 2015.

[15] K. Reich, R. Mössner, I. R. König, G. Westphal, A. Ziegler, and C. Neumann, "Promoter polymorphisms of the genes encoding tumor necrosis factor- $\alpha$ and interleukin- $1 \beta$ are associated with different subtypes of psoriasis characterized by early and late disease onset," Journal of Investigative Dermatology, vol. 118, no. 1, pp. 155-163, 2002. 
[16] P. Nikamo, S. Cheuk, J. Lysell et al., "Genetic variants of the IL22 promoter associate to onset of psoriasis before puberty and increased IL-22 production in T cells," Journal of Investigative Dermatology, vol. 134, no. 6, pp. 1535-1541, 2014.

[17] R. B. Warren, R. L. Smith, E. Flynn et al., "A systematic investigation of confirmed autoimmune loci in early-onset psoriasis reveals an association with IL2/IL21," British Journal of Dermatology, vol. 164, no. 3, pp. 660-664, 2011.

[18] C. Ronpirin, T. Tencomnao, and J. Wongpiyabovorn, "Association between the $-1438 \mathrm{~A} / \mathrm{G}$ polymorphism of the serotonin $2 \mathrm{~A}$ receptor gene and late-onset psoriasis in a Thai population," Genetics and Molecular Research, vol. 9, no. 1, pp. 208-214, 2010.

[19] U. Hüffmeier, J. Lascorz, T. Becker et al., "Characterisation of psoriasis susceptibility locus 6 (PSORS6) in patients with early onset psoriasis and evidence for interaction with PSORS1," Journal of Medical Genetics, vol. 46, no. 11, pp. 736-744, 2009.

[20] B. Nedoszytko, A. Szczerkowska-Dobosz, M. Zabłotna, J. Gleń, K. Rębała, and J. Roszkiewicz, "Associations of promoter region polymorphisms in the tumour necrosis factor- $\alpha$ gene and earlyonset psoriasis vulgaris in a northern Polish population," British Journal of Dermatology, vol. 157, no. 1, pp. 165-167, 2007.

[21] Y. Jia, H. J. Qin, J. X. Zhang, X. L. Liu, and L. J. Li, "Association of the tumour necrosis factor- $\alpha$ polymorphisms rs361525 and rs1800629 with susceptibility to psoriasis: a meta-analysis," Clinical and Experimental Dermatology, vol. 38, no. 8, pp. 836844, 2013.

[22] K. Reich, G. Westphal, T. Schulz et al., "Combined analysis of polymorphisms of the tumor necrosis factor- $\alpha$ and interleukin10 promoter regions and polymorphic xenobiotic metabolizing enzymes in psoriasis," Journal of Investigative Dermatology, vol. 113, no. 2, pp. 214-220, 1999.

[23] T. Hohler, A. Kruger, P. M. Schneider et al., "A TNF-alpha promoter polymorphism is associated with juvenile onset psoriasis and psoriatic arthritis," Journal of Investigative Dermatology, vol. 109, no. 4, pp. 562-565, 1997.

[24] F. Long, C. Sun, D. Deng, X. Zhou, X.-P. Li, and Y.-P. Zhang, "TNF-238A is associated with juvenile onset psoriasis in patients of Han population in Southwest China," Journal of Dermatological Science, vol. 36, no. 2, pp. 109-111, 2004.

[25] X. Zhang, Z. Fei, J. Wan, J. Xu, B. Yu, and M. Guan, "Association analysis of BANK1 gene with psoriasis in Southern Han Chinese," International Journal of Immunogenetics, vol. 38, no. 6, pp. 507-512, 2011.

[26] J. Wu, F. Chen, X. Zhang et al., "Association of MIF promoter polymorphisms with psoriasis in a Han population in northeastern China," Journal of Dermatological Science, vol. 53, no. 3 , pp. 212-215, 2009.

[27] J. Wongpiyabovorn, N. Hirankarn, K. Ruchusatsawat, S. Yooyongsatit, P. Asawanonda, and Y. Poovorawan, "Association of the interleukin-10 distal promoter (-2763A/C) polymorphism with late-onset psoriasis," Clinical and Experimental Dermatology, vol. 33, no. 2, pp. 186-189, 2008.

[28] N. M. Craven, C. W. Jackson, B. Kirby et al., "Cytokine gene polymorphisms in psoriasis," British Journal of Dermatology, vol. 144, no. 4, pp. 849-853, 2001.

[29] W. Weger, A. Hofer, P. Wolf et al., "The angiotensin-converting enzyme insertion/deletion and the endothelin -134 3A/4A gene polymorphisms in patients with chronic plaque psoriasis," Experimental Dermatology, vol. 16, no. 12, pp. 993-998, 2007.

[30] S. Barile, E. Medda, L. Nisticò et al., "Vascular endothelial growth factor gene polymorphisms increase the risk to develop psoriasis," Experimental Dermatology, vol. 15, no. 5, pp. 368-376, 2006.

[31] Y. T. Chang, Y. M. Shiao, P. J. Chin et al., "Genetic polymorphisms of the HCR gene and a genomic segment in close proximity to HLA-C are associated with patients with psoriasis in Taiwan," British Journal of Dermatology, vol. 150, no. 6, pp. 1104-1111, 2004.

[32] X. Solé, E. Guinó, J. Valls, R. Iniesta, and V. Moreno, "SNPStats: a web tool for the analysis of association studies," Bioinformatics, vol. 22, no. 15, pp. 1928-1929, 2006.

[33] R. Iniesta, E. Guinó, and V. Moreno, "Statistical analysis of genetic polymorphisms in epidemiological studies," Gaceta Sanitaria, vol. 19, no. 4, pp. 333-341, 2005.

[34] J. R. González, L. Armengol, X. Solé et al., "SNPassoc: an R package to perform whole genome association studies," Bioinformatics, vol. 23, no. 5, pp. 644-645, 2007.

[35] B.-J. Feng, L.-D. Sun, R. Soltani-Arabshahi et al., "Multiple loci within the major histocompatibility complex confer risk of psoriasis," PLoS Genetics, vol. 5, no. 8, Article ID e1000606, 2009.

[36] M. Chang, Y. Li, C. Yan et al., "Variants in the 5 q31 cytokine gene cluster are associated with psoriasis," Genes and Immunity, vol. 9, no. 2, pp. 176-181, 2008.

[37] S. Das, P. E. Stuart, J. Ding et al., "Fine mapping of eight psoriasis susceptibility loci," European Journal of Human Genetics, vol. 23, no. 6, pp. 844-853, 2015.

[38] P. E. Stuart, R. P. Nair, E. ELinghaus et al., "Genome-wide association analysis identifies three psoriasis susceptibility loci," Nature Genetics, vol. 42, no. 11, pp. 1000-1004, 2010.

[39] A. Strange, F. Capon, C. C. Spencer et al., "A genome-wide association study identifies new psoriasis susceptibility loci and an interaction between HLA-C and ERAP1," Nature Genetics, vol. 42, no. 11, pp. 985-990, 2010.

[40] Y. Li, W. Liao, M. Chang et al., "Further genetic evidence for three psoriasis-risk genes: ADAM33, CDKAL1, and PTPN22," Journal of Investigative Dermatology, vol. 129, no. 3, pp. 629-634, 2009.

[41] M. H. Allen, H. Ameen, C. Veal et al., "The major psoriasis susceptibility locus PSORS1 is not a risk factor for late-onset psoriasis," Journal of Investigative Dermatology, vol. 124, no. 1, pp. 103-106, 2005.

[42] S. Chantarangsu, T. Mushiroda, S. Mahasirimongkol et al., "Genome-wide association study identifies variations in 6p21.3 associated with nevirapine-induced rash," Clinical Infectious Diseases, vol. 53, no. 4, pp. 341-348, 2011.

[43] K. Douroudis, K. Kingo, H. Silm et al., "The CD226 Gly307Ser gene polymorphism is associated with severity of psoriasis," Journal of Dermatological Science, vol. 58, no. 2, pp. 160-161, 2010.

[44] T. Cabaleiro, M. Román, E. Gallo et al., "Association between psoriasis and polymorphisms in the TNF, IL12B, and IL23R genes in Spanish patients," European Journal of Dermatology, vol. 23, no. 5, pp. 640-645, 2013.

[45] W. Baran, J. C. Szepietowski, G. Mazur, and E. Baran, "A308 promoter polymorphism of tumor necrosis factor alpha gene does not associate with the susceptibility to psoriasis vulgaris. No difference either between psoriasis type I and type II patients," Acta Dermatovenerologica Alpina, Pannonica et Adriatica, vol. 15, no. 3, pp. 113-118, 2006.

[46] R. Zenz, R. Eferl, L. Kenner et al., "Psoriasis-like skin disease and arthritis caused by inducible epidermal deletion of Jun proteins," Nature, vol. 437, no. 7057, pp. 369-375, 2005. 


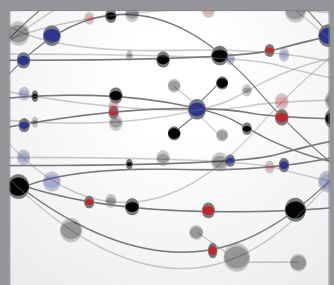

The Scientific World Journal
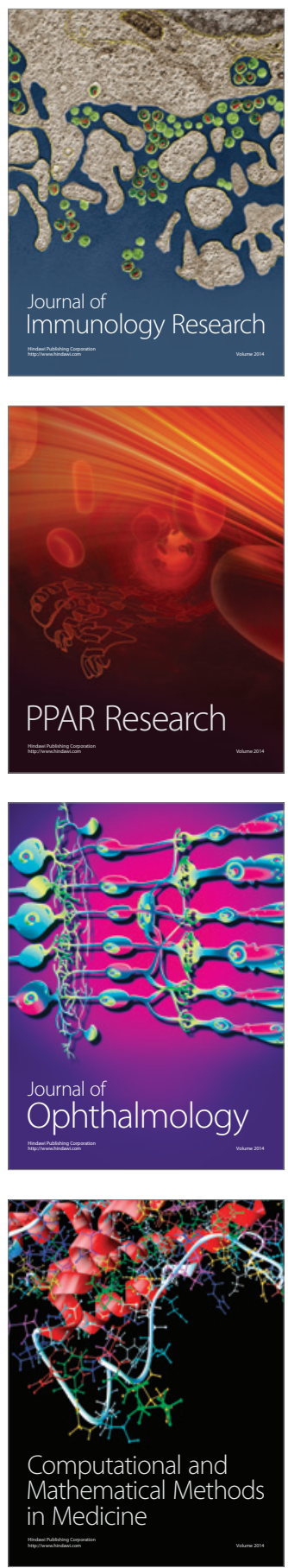

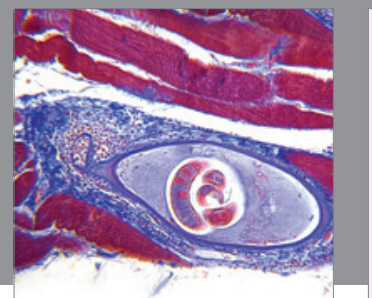

Gastroenterology

Research and Practice
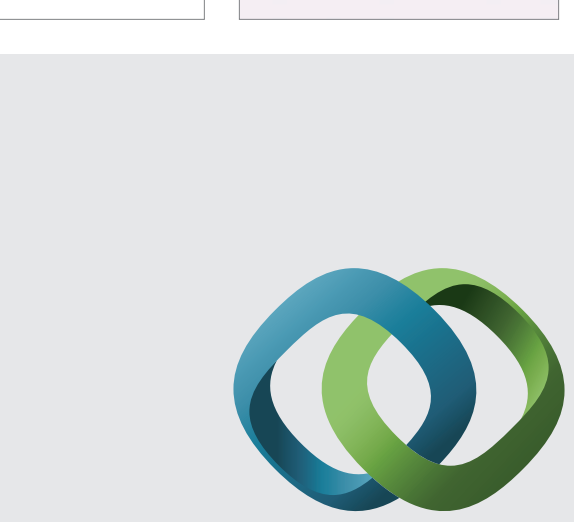

\section{Hindawi}

Submit your manuscripts at

http://www.hindawi.com
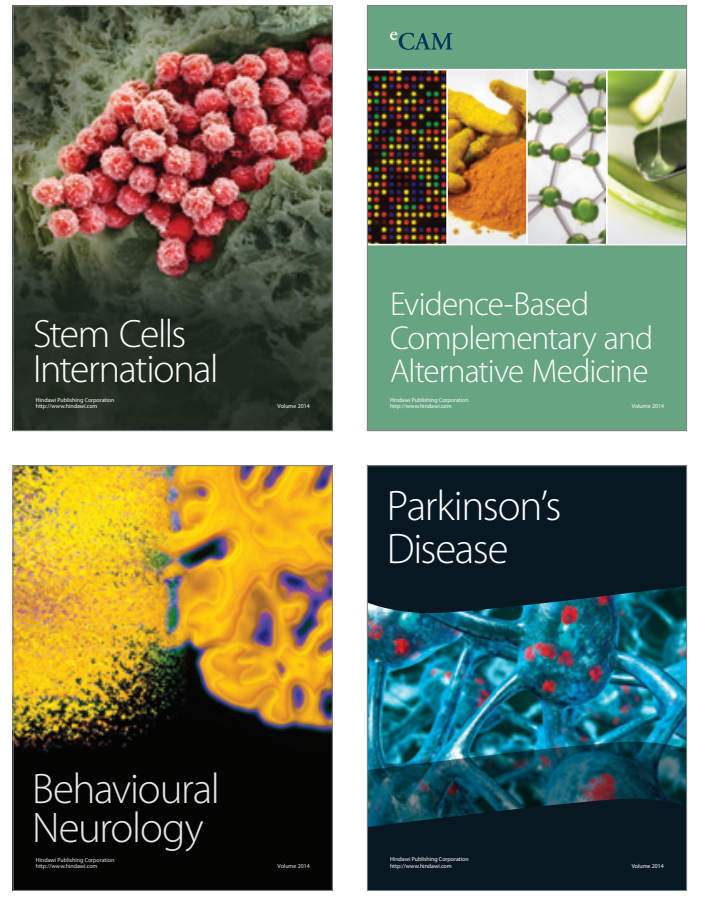
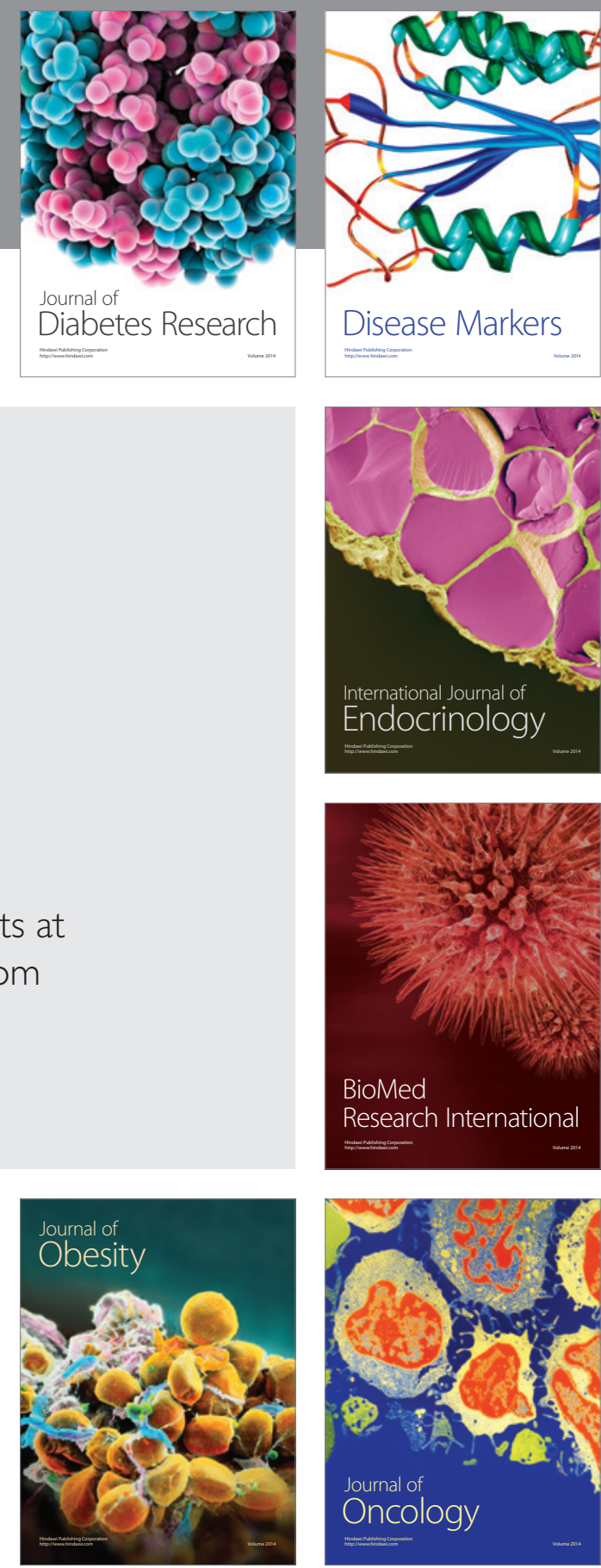

Disease Markers
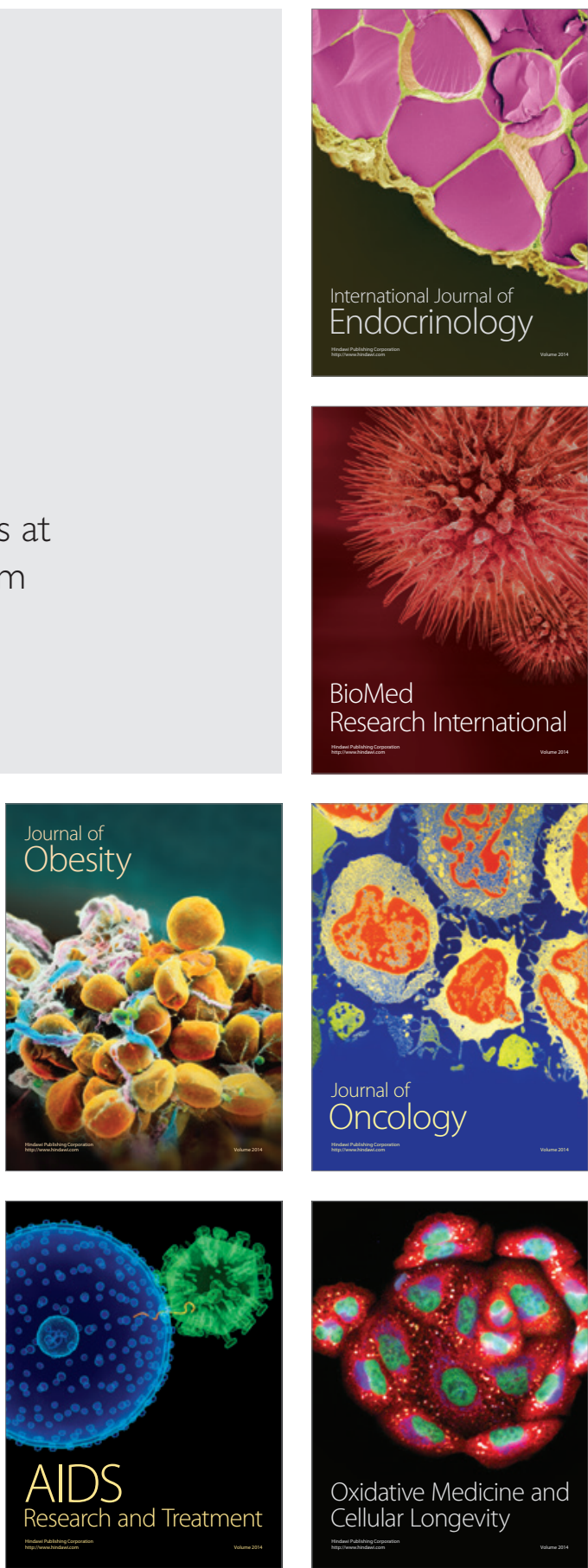